\title{
A review of microwave-induced thermoacoustic imaging: Excitation source, data acquisition system and biomedical applications
}

\author{
Yongsheng Cui, Chang Yuan and Zhong $\mathrm{Ji}^{*}$ \\ MOE Key Laboratory of Laser Life Science and Institute of Laser Life Science \\ College of Biophotonics, South China Normal University \\ Guangzhou 510631, P. R. China \\ *jizhong@scnu.edu.cn
}

Received 29 March 2017

Accepted 24 May 2017

Published 27 June 2017

\begin{abstract}
Microwave-induced thermoacoustic imaging (TAI) is a noninvasive modality based on the differences in microwave absorption of various biological tissues. TAI has been extensively researched in recent years, and several studies have revealed that TAI possesses advantages such as high resolution, high contrast, high imaging depth and fast imaging speed. In this paper, we reviewed the development of the TAI technique, its excitation source, data acquisition system and biomedical applications. It is believed that TAI has great potential applications in biomedical research and clinical study.
\end{abstract}

Keywords: Thermoacoustic imaging; microwave; biomedical applications.

\section{Introduction}

Medical imaging is a necessary tool in medical practice. Among them, X-ray imaging including CT is the clinical gold standard technique to detect abnormal structure. Nonetheless, the ionizing radiation damage limits its applications. Other imaging techniques (such as magnetic resonance imaging and ultrasound imaging) suffer from low specificity or low contrast in images and are only sensitive for selected populations. Optical imaging has high resolution and high contrast, but it suffers from low imaging depth. Therefore, it is urgent to develop a new technique that can surmount these difficulties.

As an emerging medical imaging method, microwave-induced thermoacoustic imaging (TAI), which combines the advantages of both high imaging contrast from electromagnetic absorption and high resolution of ultrasound imaging, can achieve submillimeter resolution at decimeter depth. Kruger et al. ${ }^{1-3}$ and Wang et al. ${ }^{4-27}$ open up this field almost at the same time. With the publication of above reports, more and more researchers are aware of the advantages of TAI and put their efforts into

*Corresponding author.

This is an Open Access article published by World Scientific Publishing Company. It is distributed under the terms of the Creative Commons Attribution 4.0 (CC-BY) License. Further distribution of this work is permitted, provided the original work is properly cited. 
improving TAI system. Nowadays, the main TAI groups in this field are Patch et al., ${ }^{28-33}$ Ntziachristos et al., ${ }^{34-37}$ Jiang et al., ${ }^{38-43}$ Xin et al., ${ }^{44-49}$ Arbabian et al. ${ }^{50,51}$ Zheng et al. ${ }^{52-54}$ Xing et al..$^{80-98}$ and others. ${ }^{55-79}$ Under the efforts of these researchers, the excitation source, data acquisition system and image algorithm have been improved greatly, and it can be revealed that TAI is efficient in detecting the area with anomalous microwave absorption in biological tissue, particularly for breast cancer. This paper will review and discuss the TAI system briefly and focus on the introduction of excitation source, data acquisition system and biomedical applications.

\section{The Principle}

When a microwave pulse irradiates to biological tissue, a small temperature rise can be produced, and the heated tissue will expand and shrink into an acoustic source. When microwave energy delivered in a pulse is short enough so that thermal diffusion can be ignored during the pulse, the resulting thermoacoustic pressure $P(r, t)$ that reaches a detector at position $r$ and time $t$ can be expressed as the following equation ${ }^{1-3}$ :

$$
P(r, t) \approx \frac{\beta I_{0} C_{0}}{4 \pi C_{p}} \tau \frac{\mathrm{d}}{\mathrm{d} t} \oiint_{\left|r-r^{\prime}\right|=C_{0} t} A\left(r^{\prime}\right) \frac{d r^{\prime}}{C_{0} t},
$$

where $\beta$ is the isobaric volume expansion coefficient, $I_{0}$ a factor proportional to the incident optical energy density, $C_{0}$ the speed of ultrasound, $C_{p}$ the specific heat, $\tau$ the microwave pulse width and $A\left(r^{\prime}\right)$ the absorbed energy density per unit volume of soft tissue at position $r^{\prime}$ at time $t$. The induced thermoacoustic wave propagates from its originating region and can be detected by an ultrasonic detector. The strength and the profile of a thermoacoustic signal depend on the microwave absorption of the tissue.

Although sharing the similar principle with the photoacoustic imaging (PAI), TAI may have broader applications in medical imaging. The effect of microwave on biological tissue is mainly reflected in the electric field, and electrical field at the depth $z$ can be expressed as:

$$
E=E_{0} \exp (-\alpha z),
$$

where $E$ is the electrical field at depth $z, E_{0}$ the electrical field at the sample surface and $\alpha$ the electric field absorption coefficient expressed as:

$$
\alpha=\omega \sqrt{\frac{\mu \varepsilon}{2}\left(\sqrt{1+\left(\frac{\sigma}{\omega \varepsilon}\right)^{2}}-1\right)},
$$

where $\omega$ is the angular frequency, $\mu$ the permeability, $\varepsilon$ the permittivity and $\sigma$ the conductivity. The induced acoustic pressure is expected to depend on the intensity of microwave and the dielectric constant of the material. In the frequency range from 0.1 to $10 \mathrm{GHz}$, the relative dielectric constant has a value of 5-70 for soft tissues, and the conductivity has a value of $0.02-3 \mathrm{~S} / \mathrm{m}$. The dielectric properties of tissue determine the absorption of microwave at various frequencies. For example, at $9.4 \mathrm{GHz}$, the penetration depth, which is the inverse of the absorption coefficient, is 3.4 and $0.35 \mathrm{~cm}$ for fat and muscle; whereas at $500 \mathrm{MHz}$, the penetration depths for fat and muscle are 23.5 and $3.4 \mathrm{~cm}$. Most of the other soft tissues have an absorption coefficient between muscle and fat. Moreover, as long as microwave energy reaches the target, the thermoacoustic effect will be generated. Therefore, TAI may potentially be used to detect the deep object with anomalous microwave absorption.

\section{The Excitation Source}

TAI and PAI technology are based on the thermoelastic effect, and the main difference is the excitation source. TAI uses microwave as the excitation source, and PAI uses laser. Because of high penetration depth of microwave, TAI obtains a good imaging depth, generally at $6-15 \mathrm{~cm}$. However, the microwave beam is hard to focus due to diffraction, so that the existing excitation sources have highpower outputs, mainly divided into three types, namely, magnetron modulation mode, high-voltage discharged mode and modulated continuous mode.

\subsection{Magnetron modulation mode}

Microwave generator in magnetron modulation mode is usually composed of electron emitter, power supply, magnetron and waveguide, as shown in Fig. 1. The electrons from electron emitter can be accelerated and generate stable electronic oscillation in magnetron. Then the electronic oscillation can be transferred to microwave in the waveguide. At the end of waveguide, microwave transforms from waveguide pattern to radiation pattern due to 


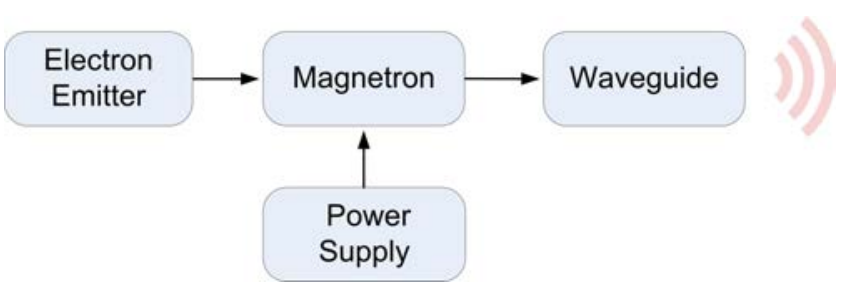

Fig. 1. Schematic diagram of magnetron modulation mode.

diffraction. As oscillation in magnetron is stable and the waveguide has a frequency-selective effect, microwave in this situation has a single frequency and be suitable for mapping absorption coefficient. At the meantime, sample under test is usually placed at the end of the waveguide, where energy is relatively concentrated, so that a better signal-to-noise ratio (SNR) can be obtained. Therefore, most researchers including Wang et al., Jiang et al., Xin et al., Patch et al., Ntziachristos et al. and Xing et al. adopted waveguide mode. Details can be seen in Table 1 . Figure 2(a) shows a typical microwave generator working in the waveguide mode and Fig. 2(b) depicts several pulse widths of this microwave generator.

However, those microwave generators have two weaknesses. One is that the main frequency cannot change due to the fixed operating frequency of magnetron and waveguide, i.e., against selective excitation. The other is that the pulse width is difficult to narrow due to manufacturing technology of magnetron, and the minimum is commonly
$0.2-0.3 \mu \mathrm{s}$, which limits imaging resolution to about $0.5 \mathrm{~mm}$.

\subsection{High-voltage discharged mode}

In order to get a narrower pulse width and to improve imaging resolution, microwave in highvoltage discharged mode has been adopted. As is shown in Fig. 3, the microwave generator is usually composed of oscillation circuit, boosting transformer, high-speed switch and antenna. Similar to the magnetron, the electrons can form electrical pulses in the oscillation circuit and then boost to high voltage by transformer. Finally, the highvoltage pulse propagates through the antenna into unbounded medium, namely, microwave. The pulse width of microwave can reach a few nanoseconds depending on the speed of the switch, so that the resolution can realize about dozens of microns' range. Ntziachristos et al. and Xing et al. adopted microwave in this mode, and the details can be seen in Table 1.

Especially, the ultrashort microwave pulse (USMP) by Xing et al. successfully demonstrated a means of overcoming the conversion efficiency and resolution limited by the exciting pulse duration. The setup developed for experimental validation is schematically shown in Fig. 4(a). The core of the system is a USMP generator that is based on highvoltage-pulsed power technology. The USMP generator makes use of a charging circuit and Tesla

Table 1. Comparison of microwave sources by research groups.

\begin{tabular}{lcccc}
\hline Research group & Pulsed width & Main frequency & Producing mode & Peak power \\
\hline R. A. Kruger & $0.5 \mu \mathrm{s} / 1.0 \mathrm{~ms}^{1-3}$ & $434 \mathrm{MHz}$ & $\mathrm{HD}$ & $25 \mathrm{KW}$ \\
V. Ntziachristos & $10 \mathrm{~ns}^{34}$ & $\sim 100 \mathrm{MHz}$ & $\mathrm{HD}$ & $\sim 70 \mathrm{MW}$ \\
& $10 / 15 / 45 \mathrm{~ns}^{36}$ & $\sim 400 \mathrm{MHz}$ & $\mathrm{HD}$ & $2.5 / 3.3 / 5.8 \mathrm{MW}$ \\
D. Xing & $10 \mathrm{~ns}^{88-91}$ & $434 \mathrm{MHz}$ & $\mathrm{HD}$ & $4-40 \mathrm{MW}$ \\
& $0.5 \mu \mathrm{s}^{79-82}$ & $1.2 \mathrm{GHz}$ & $\mathrm{MM}$ & $300 \mathrm{KW}$ \\
& $0.3-1.1 \mu \mathrm{s}^{83-87}$ & $6 \mathrm{GHz}$ & $\mathrm{MM}$ & $350 \mathrm{KW}$ \\
L. V. Wang & $0.6-2.2 \mu^{14}$ & $9.4 \mathrm{GHz}$ & $\mathrm{MM}$ & $10 \mathrm{KW}$ \\
& $0.5 \mu^{5,7,8}$ & $3 \mathrm{GHz}$ & $\mathrm{MM}$ & $2 \mathrm{KW}$ \\
H. Jiang & $0.5 \mu^{6}$ & $9 \mathrm{GHz}$ & $\mathrm{MM}$ & $25 / 10 \mathrm{KW}$ \\
H. Xin & $0.75 \mu \mathrm{s}^{39-41}$ & $3 \mathrm{GHz}$ & $\mathrm{MM}$ & $70-100 \mathrm{KW}$ \\
& $400 \mathrm{~ns}^{44}$ & $2.7-3.1 \mathrm{GHz}$ & $\mathrm{MC}$ & $5.2 \mathrm{KW}$ \\
A. Arbabian & $0.5-1.5 \mu \mathrm{s}^{45-49}$ & $3 / 1.4 / 2.5 \mathrm{GHz}$ & $\mathrm{MM}$ & $4 / 10 /-\mathrm{KW}$ \\
S. K. Patch & $\mathrm{NA}$ & $2 / 2.1 \mathrm{GHz}{ }^{50,61}$ & $\mathrm{MC}$ & $120 \mathrm{~W}$ \\
Y. Zheng & $700 \mathrm{~ns}^{31,32}$ & $108 \mathrm{MHz}$ & $\mathrm{MM}$ & $20 \mathrm{KW}$ \\
\hline
\end{tabular}

Note: HD: High-voltage discharged mode; MM: Magnetron modulation mode;

MC: Modulated continuous mode; NA: Not available. 
Y. Cui, C. Yuan \& Z. Ji

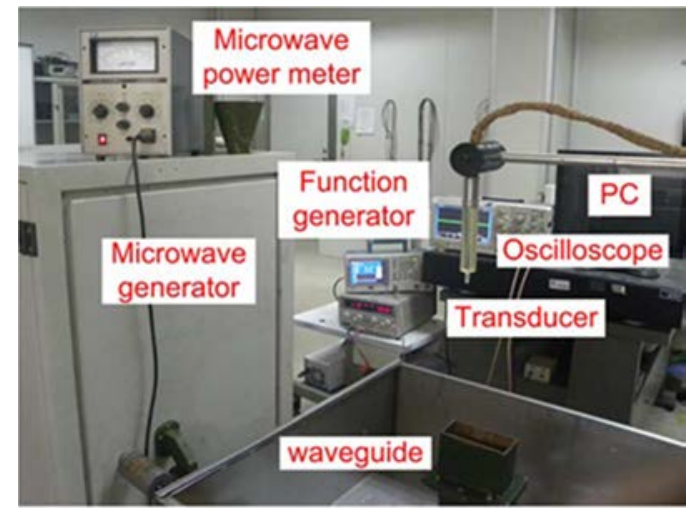

(a)

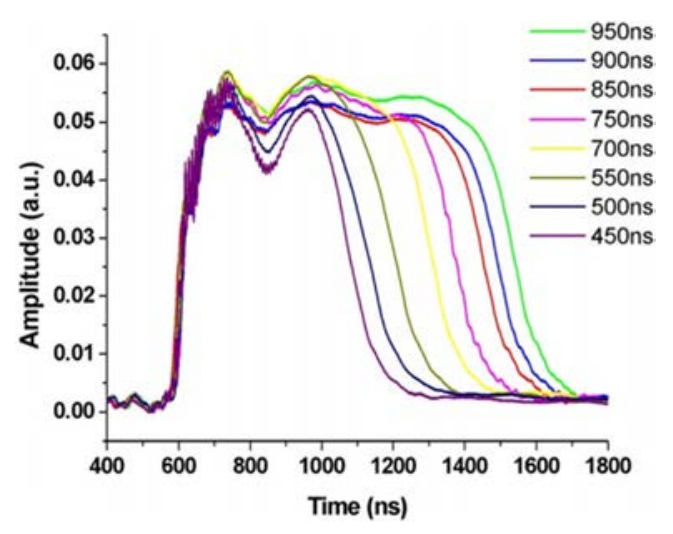

(b)

Fig. 2. (a) A typical microwave generator working in the waveguide mode. ${ }^{92}$ (b) Several pulse widths of this microwave generator. $^{86}$

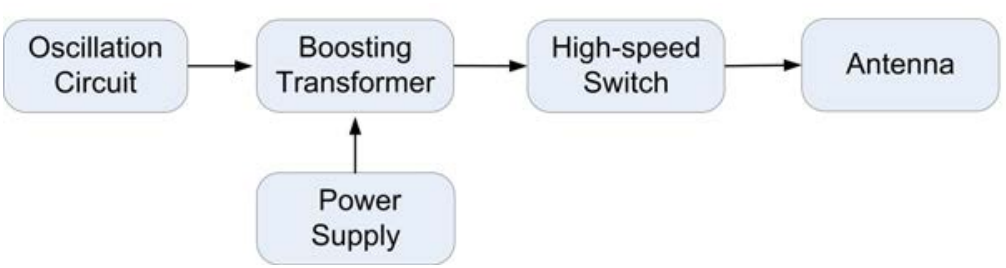

Fig. 3. Schematic diagram of high-voltage discharged mode.

transformer to produce high-voltage pulses and then discharge to the antenna for radiating microwave. The USMP generator has features of short pulse width, high peak power, simple structure and easy miniaturization. The pulse duration is measured to be $10 \mathrm{~ns}$ with $800 \mathrm{ps}$ rise time, and the peak output power is tunable from 2 to $40 \mathrm{MW}$, which satisfies the need of TAI.

To investigate the spatial resolution of the USMP-induced TAI, the cross-sections of two

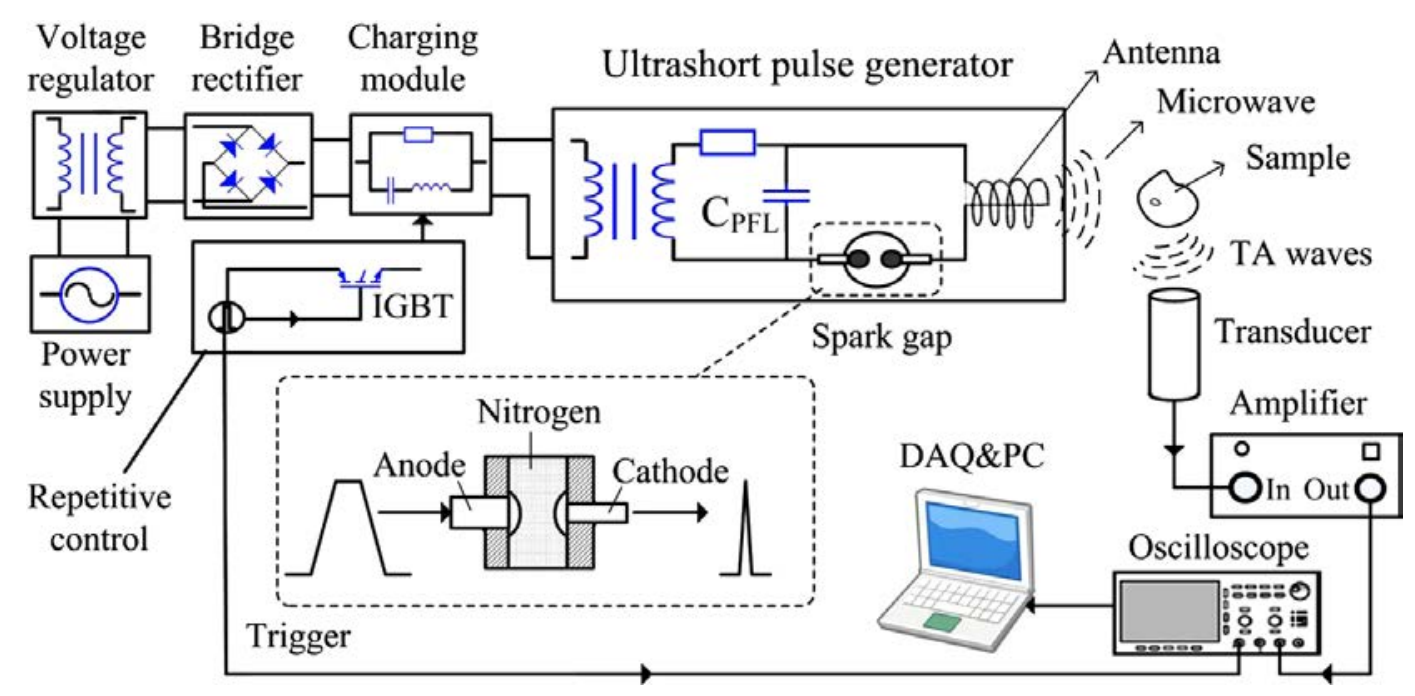

(a)

Fig. 4. (a) Schematic diagram of the experimental setup of USMP TAI. (b) The comparison of microwave pulses of the USMP and the microsecond-level TAI. (c) The microwave pulse of the USMP. ${ }^{88}$ 


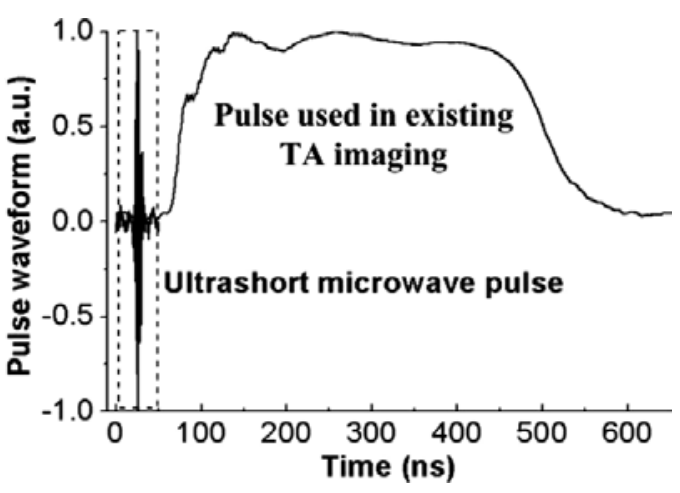

(b)

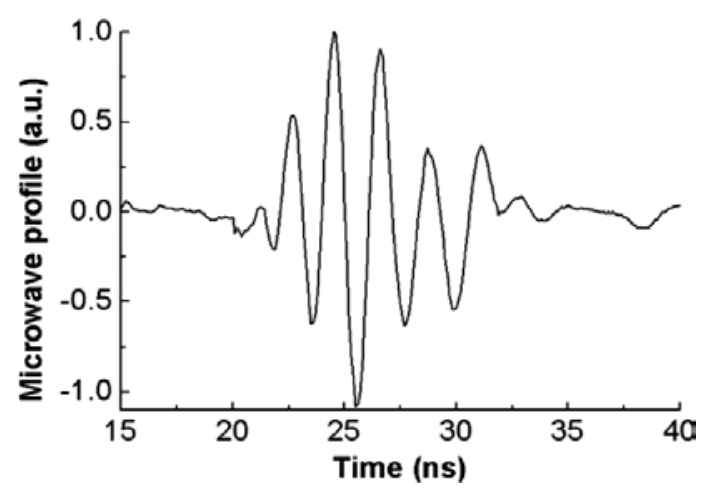

(c)

Fig. 4. (Continued)

copper wires were imaged, as shown in Fig. 5. In the reconstruction image in Fig. 5(a), the two wires can be clearly detected. Figure 5(b) plots the normalized intensity profile of the cross-section image, and

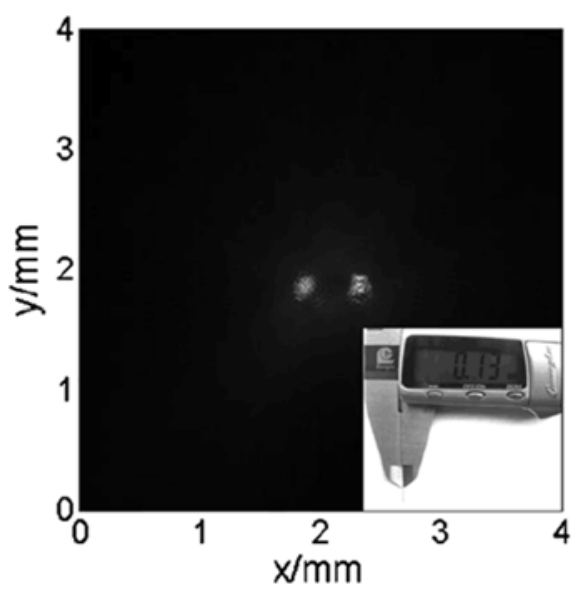

(a)

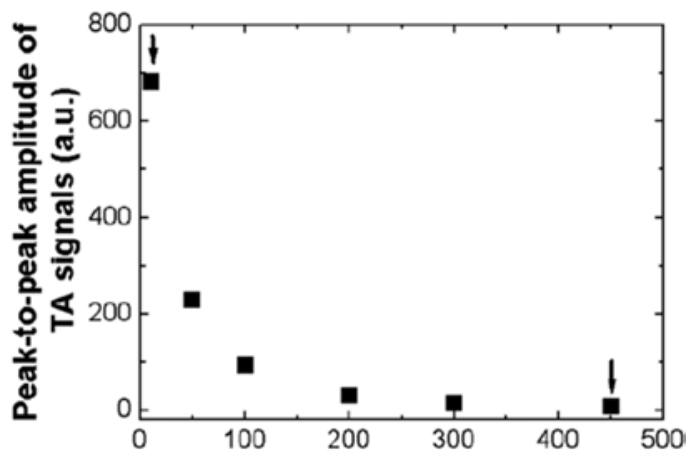

(c) the spatial resolution is determined to be $105 \mu \mathrm{m}$ according to the Rayleigh criterion. More importantly, the TA conversion efficiency was compared with short- and long-pulse (microsecond-level)

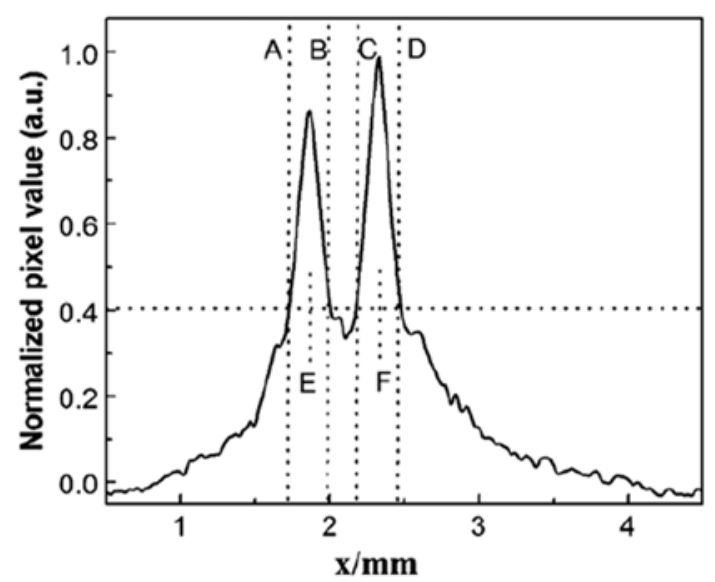

(b)

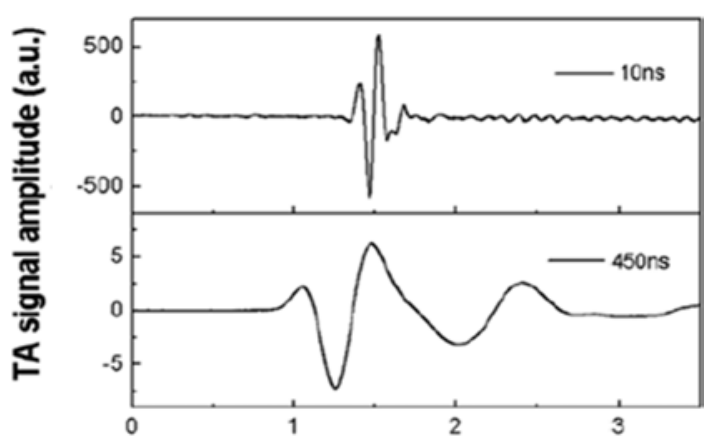

(d)

Fig. 5. Spatial resolution of the USMP TAI system. (a) TAI of two copper wires $(0.13 \mathrm{~mm}$ in diameter). The inset figure at the bottom-right corner shows the diameter of the wire. (b) Line profile of the reconstructed image shown in (a) with $y=1.84$ mm. (c) The simulated peak-to-peak amplitude of TAI signal of the USMP and the microsecond-level TAI. (d) The experimental results of TAI signal amplitude. ${ }^{88}$ 
excitation. Figure 5(c) gives the simulation results of the amplitude of the TAI signal, which was excited by various microwave pulse durations with identical pulse energy. Also, the experimental results in Fig. 5(d) show the amplitude of the TAI signal obtained by using a $10 \mathrm{~ns}$ microwave, which is 86-fold greater than that generated by a microwave pulse with duration of $450 \mathrm{~ns}$. Therefore, it is expected that the enhancement of TA excitation efficiency should be about two orders of magnitudes.

However, some disadvantages still exist. One is that the frequency of the microwave limits to low frequency $(100-900 \mathrm{MHz})$ and wide bandwidth $(\sim 100 \mathrm{MHz})$. At low frequency, microwave absorption coefficient is relatively small, which may be beneficial to imaging depth, but result in low SNR. Meanwhile, due to poor directivity of antenna, the energy scatters in whole space, resulting in a lower SNR, limiting its biomedical application.

\subsection{Modulated continuous mode}

The above two TAI modes adopt pulsed microwave, and the main frequency and pulse width are difficult to change. Using modulated continuous microwave can solve the problem. As is shown in Fig. 6, the microwave generator is usually composed of signal source, modulator, power amplifier (PA) and antenna. The signal source can generate a low-power signal with variable frequency, and then the signal is modulated to pulsed mode by modulator. After $\mathrm{PA}$, the high-power pulse transforms into unbounded medium propagation by antenna. Because the frequency is changed by signal source and modulator, the main frequency and pulse width are continuously adjustable. Therefore, this scheme has a wide range of applications, especially in material properties testing. However, the power of that microwave is restricted by $\mathrm{PA}$, and its long-imaging time limits clinical application.

Zheng et al. used modulated continuous microwave to observe both TA and thermoacoustic resonance (TAR) effect, as shown in Fig. 7(a). A microwave generator (SMBV100A, Rohde \& Schwarz) generates single and multi-pulse microwave source for the observation of TA and TAR effects, respectively. The microwave source is amplified up to $100 \mathrm{~W}$ with a microwave PA

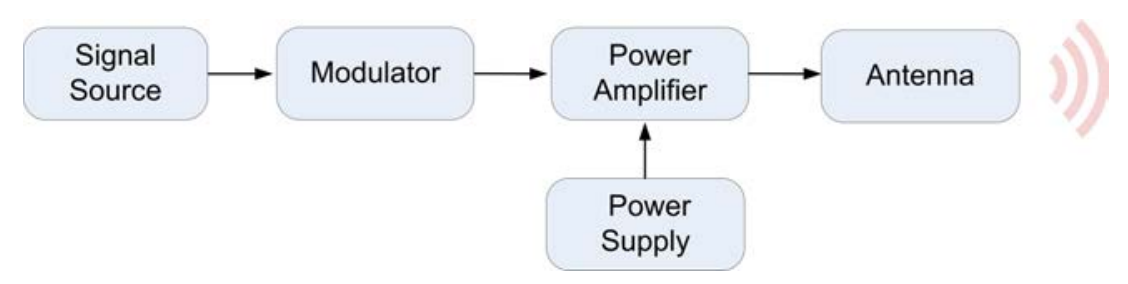

Fig. 6. Schematic diagram of modulated continuous mode.

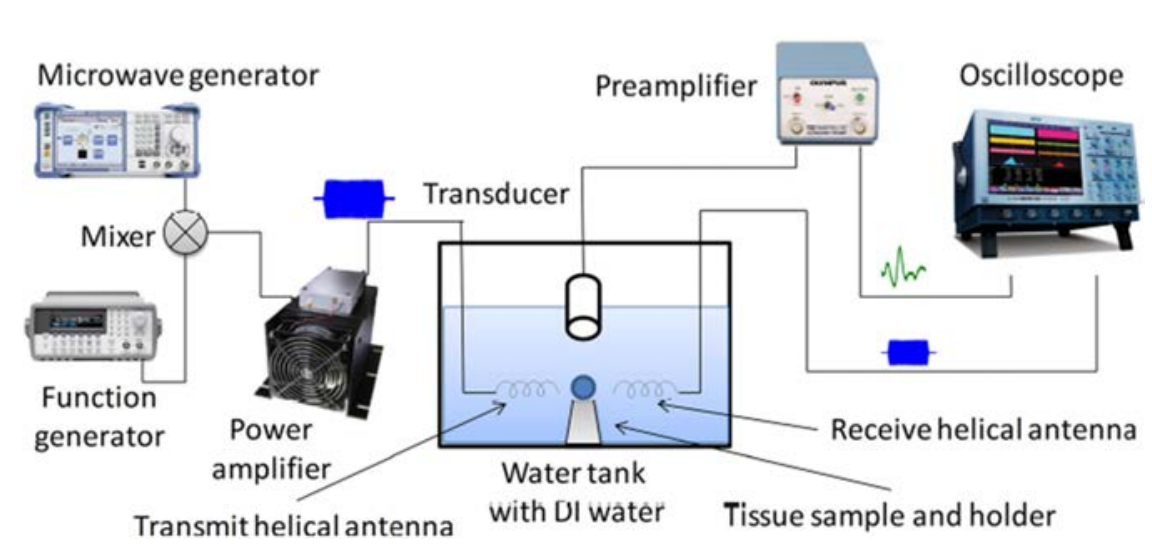

(a)

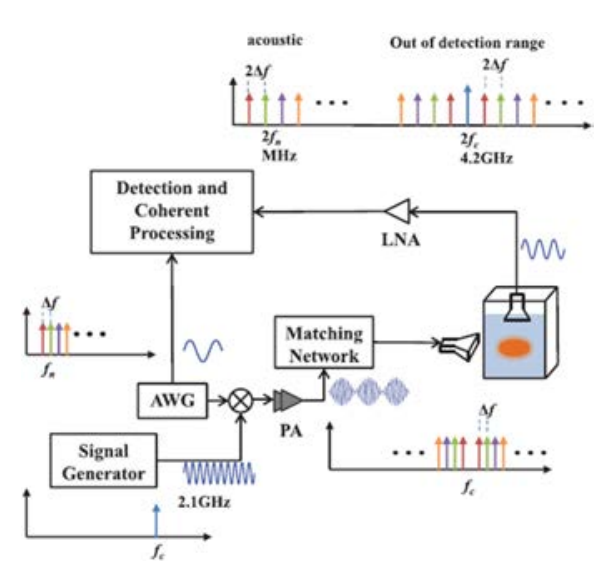

(b)

Fig. 7. (a) Schematic diagram of the experimental setup for the observation of both TA and TAR effect induced by single-pulse and multi-pulse microwave source. (b) The schematic of the experiment setup. The AWG sends out the stepped-frequency envelope signal that modulates the microwave carrier. ${ }^{55}$ 
(ZHL-100 W-GAN+, Mini-Circuits). Considering the tissue absorption characteristics with respect to microwave frequencies, $440 \mathrm{MHz}$ was chosen as the carrier frequency. The pulsed microwave signal is then fed into a custom-designed helical antenna operating at $440 \mathrm{MHz}$. By this system, they successfully generate detectable TAI signal.

Also, Arbabian used a stepped-frequency continuous-wave microwave for TAI, as shown in Fig. 7(b). The arbitrary wave generator (AWG) generates the low-frequency stepped-frequency envelope signal that then modulates a $2.1 \mathrm{GHz}$ carrier. A gallium nitride PA is designed to bring the peak power up to $120 \mathrm{~W}$ (for pulse operation). Unlike other solutions that employ KW sources, the solution has a significantly lower peak power and is implemented completely with solid-state electronics. The pulse width is $1 \mu \mathrm{s}$ and the microwave frequency is at $2.1 \mathrm{GHz}$. In the experiments, a planar immersion piezoelectric transducer with a central frequency of $0.5 \mathrm{MHz}$ is used for detection. A low noise amplifier is employed for conditioning. After a low pass filter, the signal is averaged (typically 1000 times) and sampled by an oscilloscope. A linear stage setup is used to perform a B-Scan to construct the image of the sample.

\section{Data Acquisition System}

The equipment of ultrasound imaging technology, such as ultrasonic transducer, can be used in the detection of TAI signals, so that TAI has the similar resolution of ultrasound imaging. At present, the ultrasonic transducers are made of piezoelectric crystal materials, polyvinylidene fluoride (PVDF) film and piezoelectric ceramic. Piezoelectric crystal materials can provide simple, accurate and high sensitivity of the signal detection. PVDF thin film material has the advantages of low acoustic impedance and frequency bandwidth, but its sensitivity is relatively low. Piezoelectric ceramic material has high sensitivity, but the bandwidth is narrow. The common acoustic detectors are divided into single-element and multi-element detectors, according to the number of elements. In accordance with the different detectors, the detection system is generally divided into single-element and multielement detection systems, as shown in Table 2.

\subsection{Scanning mode}

The circular-scanning (CS) and linear-scanning (LS) modes are usually used in traditional TAI systems, as shown in Fig. 8. The CS mode was employed in most of the research on TAI techniques, which requires a single-element transducer rotating around the sample in circular fashion to acquire complete information of the target. A typical work flow of the single-element TAI system is shown in Fig. 9. The tissue absorbs microwave energy and then generates TA signals, and the singleelement detector is placed at a distance from the center of the sample to receive the TAI signal. The received signal is amplified to the oscilloscope (which can also be replaced by a data acquisition card). In the oscilloscope, the TA signal is averaged

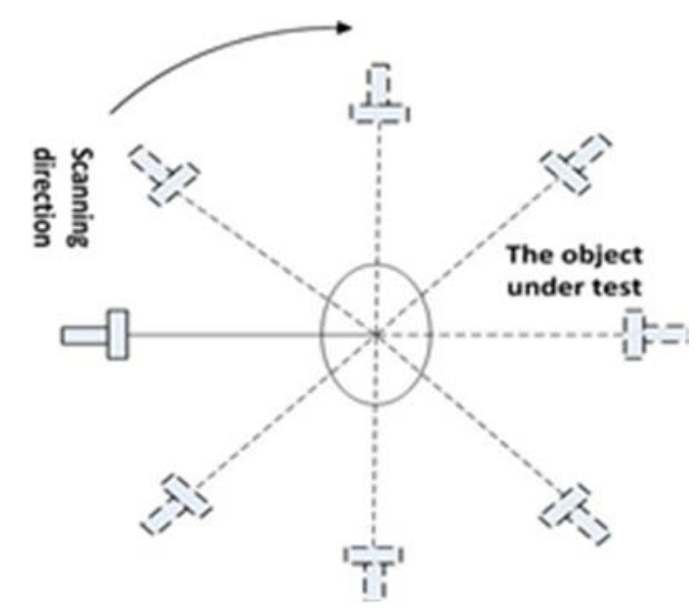

(b)

(a)

Fig. 8. Schematic diagram of LS (a) and CS (b). 
Y. Cui, C. Yuan \& Z. Ji

Table 2. Comparison of DAS by research groups.

\begin{tabular}{llcccc}
\hline Research group & \multicolumn{1}{c}{ Type } & Shape & Array element & Center frequency & Resolution \\
\hline R. A. Kruger & Multi-element $^{1-3}$ & NA & 64 & $1.0 \mathrm{MHz}$ & $\sim 1 \mathrm{~mm}$ \\
V. Ntziachristos & Single-element $^{34}$ & NA & 1 & $7.5 \mathrm{MHz}$ & $170 \mu \mathrm{m}$ \\
& Single-element $^{36}$ & NA & 1 & $3.5 / 15 \mathrm{MHz}$ & $45 \mu \mathrm{m}$ \\
D. Xing & Single-element $^{82-85}$ & NA & 1 & $2.5 / 3.5 \mathrm{MHz}$ & $0.5 \mathrm{~mm}$ \\
& & Linear $^{97}$ & 128 & $2.5 \mathrm{MHz}$ & $2.2 \mathrm{~mm}$ \\
& Multi-element & Full ring $^{91}$ & 384 & $10 \mathrm{MHz}$ & NA \\
L. V. Wang & Flexible $^{94}$ & 64 & $7.5 \mathrm{MHz}$ & NA \\
& Single-element $^{4,7,8}$ & NA & 1 & $1 / 3.5 \mathrm{MHz}$ & $1.5 \mathrm{~mm}$ \\
H. Jiang & Multi-element $^{5,6}$ & Linear & 30 & $2.25 \mathrm{MHz}$ & $1.9-2.5 \mathrm{~mm}$ \\
H. Xin & Single-element $^{39-41}$ & NA & 1 & $2.25 \mathrm{MHz}$ & $500 \mu \mathrm{m}$ \\
& Single-element $^{44}$ & NA & 1 & $1 \mathrm{MHz}$ & $500 \mu \mathrm{m}$ \\
A. Arbabian & Multi-element $^{45-49}$ & Linear & 128 & $2.25 \mathrm{MHz}$ & mm order \\
S. K Patch & Single-element $^{50,51}$ & NA & NA & $0.5 \mathrm{MHz}$ & NA \\
& Single-element $^{31}$ & NA & 1 & $2.25 \mathrm{MHz}$ & NA \\
Y. Zheng & Multi-element $^{32,33}$ & Linear & 96 & $1-4 \mathrm{MHz}$ & $250 \mu \mathrm{m}$ \\
& Single-element $^{52-64}$ & NA & 1 & $\mathrm{NA}$ & NA \\
\hline
\end{tabular}

Notes: NA: Note available; DAS: Data acquisition system.

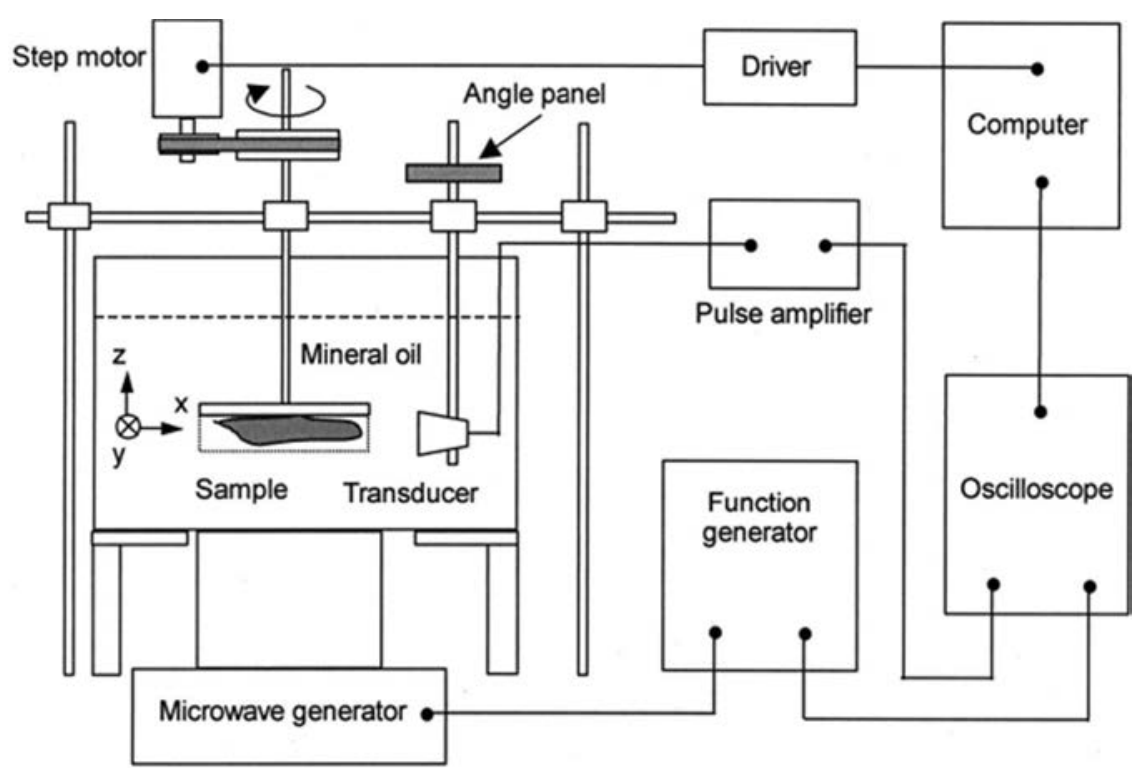

Fig. 9. Schematic diagram of single-element TAI system. ${ }^{8}$

multiple times, and then it is sent into the computer through the general interface bus. After the signal data transmission completes, the computer commands the stepper motor to drive the detector to rotate-scanning. When the detector rotates to the next position, the computer controls the acquisition system to scan signal for the next round until the $360^{\circ}$ omnidirectional scanning is completed. In addition, a 2-D image can be reconstructed by using the filtered back-projection algorithm (or other algorithm), which can reflect microwave absorption distribution of the biological tissue. Therefore, the single-element detector can acquire 2-D images by collecting signals using multi-position scanning (typically requiring dozens to hundreds of positions). Almost all groups above adopted single-element data acquisition system (DAS) at the beginning of the study, and details can be seen in Table 2 .

Although this imaging mode can well reflect the electromagnetic wave absorption distribution of biological tissue, its experimental device and the source of the error are complex, and the data 
acquisition time is long (typically $10-30 \mathrm{~min}$ ), and the computational complexity of the reconstructed image takes a long time. Therefore, the development of multi-element detection systems is needed.

\subsection{Multi-element data acquisition}

As shown in Fig. 10(a), the process of multielement data acquisition used by Xing et al. is depicted. In the imaging process, when the microwave pulses illuminate the object, the TA signals are detected by the 384 detectors simultaneously, and each group of 64 element signals passes through the switching system in sequence and is acquired by two 32-element data acquisition cards (NI5752, NI Inc., USA) at a sampling rate of $50 \mathrm{MHz}$. After switching six times, all 384 signals are acquired.
Finally, the 384-element signals are used to reconstruct a 2-D image via a filtered back-projection algorithm. Figures 10(b)-10(d) show images of the system. By the multi-element data acquisition system, the data acquisition time can reduce from dozens of minutes to $0.06 \mathrm{~s}$, realizing real-time imaging. Figure 11 shows several typical multielement detectors. Figure 11(a) is a commercial 128-element linear array transducer (SIUI Inc., L2L50A, Shantou, China). The central frequency of the transducer is $2.5 \mathrm{MHz}$ with a bandwidth of $70 \%$. Figure 11(b) shows that full ring transducer (10C384-1.62 8-R100 AHA001, Doppler Ltd, China) is made up of three parts, and every part contains 128 elements. The central frequency of the transducer is $10 \mathrm{MHz}$ and the bandwidth ranges from $64.5 \%$ to $92.4 \%$.

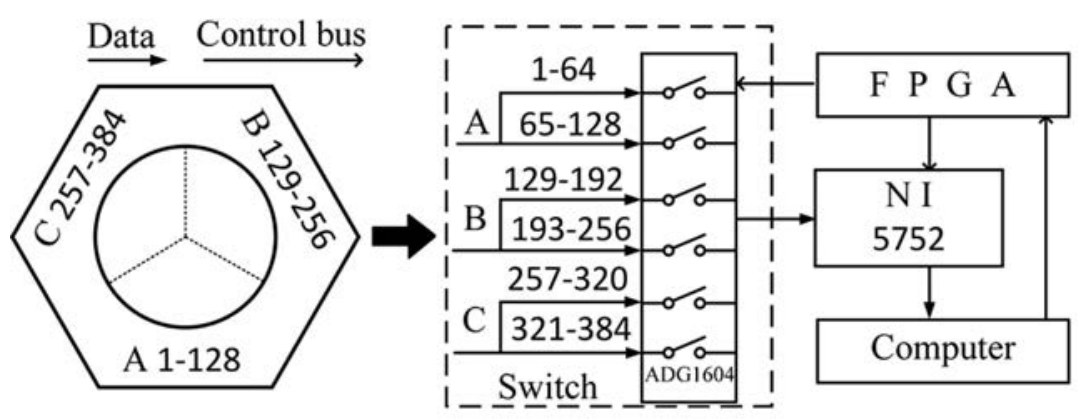

(a)

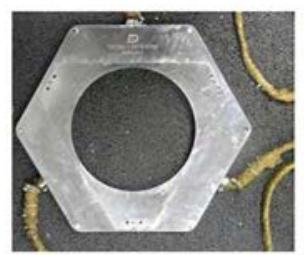

(b)

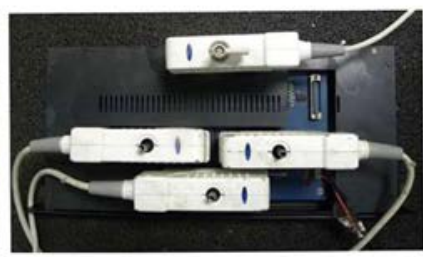

(c)

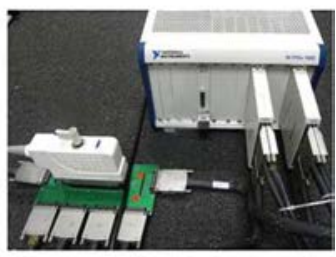

(d)

Fig. 10. (a) The process of signal acquisition of multi-element TAI system. (b) Photographs of the full ring transducer. (c) The 384-64 element switch system. (d) The 64 -element data acquisition system. ${ }^{91}$

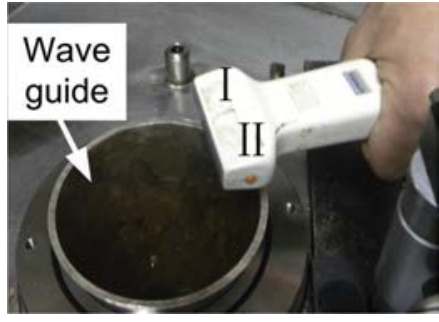

(a)

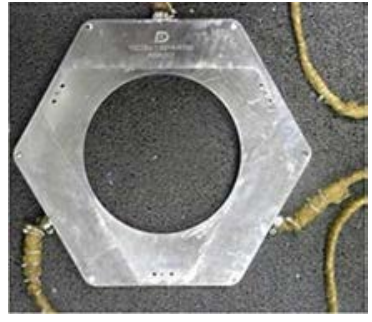

(b)

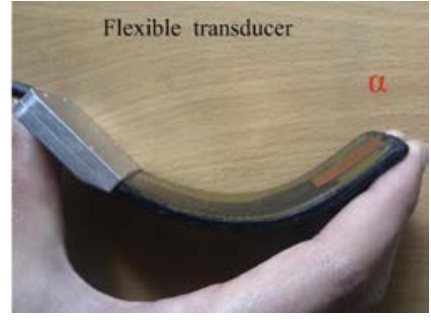

(c)

Fig. 11. Photographs of detectors. (a) Linear detector..$^{97}$ (b) Full ring detector. ${ }^{91}$ (c) Flexible detector. ${ }^{94}$ 
However, the above ring detectors employ a fixed scanning radius to acquire information from different positions. For irregular samples, the scanning radius must be large enough to cover the longest axis of the imaging geometry. Thus, a large amount of coupling liquid required to fill in the gap between sample and detector hinders the practical application of TAI. Also, the linear detector has been widely used in medical ultrasonic imaging for body scanning, although it is not suitable for TAI of irregular objects due to image distortion and low utilization. Therefore, Xing et al. present a samplecling-scanning (SCS) model that combines the advantages of both ring and linear detector. To implement this model, a flexible multi-element transducer and adaptive back projection algorithm are presented. As shown in Fig. 11(c), most of the elements in the SCS model can be brought in close contact with sample due to the controllable shape of the transducer. Theoretically, it can adapt to samples with any shape without requiring coupling liquid, especially for breast cancer detection.

In summary, the single-element system is the most primitive equipment in TAI; it has the advantage of simple and cheap, but suffers from long-imaging time and instability, so it is suitable for material research. The multi-element system is specially designed for biomedical applications, mainly for breast detection. However, it applies only to single application. Among them, the linear detector, which has been widely used in medical ultrasonic imaging for body scanning, may become universal testing equipment after modification.

\section{Biomedical Applications}

Generally speaking, TAI can detect the microwave absorption anomaly at decimeter depth with submillimeter resolution, and the application of TAI needs to meet two basic conditions. One is the diseased tissue and the normal tissues have microwave absorption difference. For example, breast cancer tissue has absorption higher than normal breast tissue, and the ratio is estimated to be about $2: 1-10: 1$. Another is the microwave energy can penetrate to the target, which can be guaranteed in humans, and the ultrasonic wave can spread out received by the detector. In particular, air and bone are against ultrasonic propagation, so anomaly within lung, human skull and bone is not appropriate to be detected.

\subsection{Early breast cancer detection}

The most important application of TAI is the early breast cancer detection. The main component of the breast is fat, which performs a low absorption in the microwave band. Therefore, in the early stages of the tumor, microwave absorption can increase significantly, due to ionic or water content growing up at the margin of the tumor. The change of absorption distribution of the tissues usually means physiological lesions, and the previous results suggest that the dielectric properties of malignant breast tissues are larger than the normal tissues. Therefore, the detection of breast cancer by TAI is feasible, and recently, more and more attention is paid to apply TAI to the detection of breast cancer, such as Kruger et al., Wang et al. and Xing et al.

Among them, Xing et al. developed a system that provides a rapid and noninvasive approach for highcontrast breast cancer imaging. The results of TAI on the malignant breast tissue are shown in Fig. 12(a). In the results of TAI, a strong contrast was observed with satisfactory consistency in tumor position and shape, and the tumor-to-background contrast was estimated to be about 5.5:1, which demonstrates that TAI has a higher imaging contrast than X-ray mammography. Later, Xing et al. used the pathology model to view imaging capabilities. The pathology model is composed of excised human breast tumor and an adult ewe, as shown in Fig. 12(b). It can be seen that the difference between the normal tissue and the tumor in the TAI has a mean contrast of about 1:2.8. Further, Xing et al. performed a 3-D TAI experiment on breast model with a human tumor, and the results are shown in Fig. 12(c). Up to now, early breast cancer detection is the application that closest to the clinical, and the development direction is to clinical practice.

\subsection{Prostate cancer}

The prostate cancer has a higher microwave absorption than the surrounding normal tissues, so it is different on the TAI. Patch et al. presented a TAI system for performing ex vivo prostate cancer sample with very high frequency energy. The system utilized irradiation pulses of $700 \mathrm{~ns}$ duration exceeding $20 \mathrm{~kW}$ power and a dual-transducer system to achieve high contrast of prostate cancer. In the reconstruction images, the verumontanum can be 

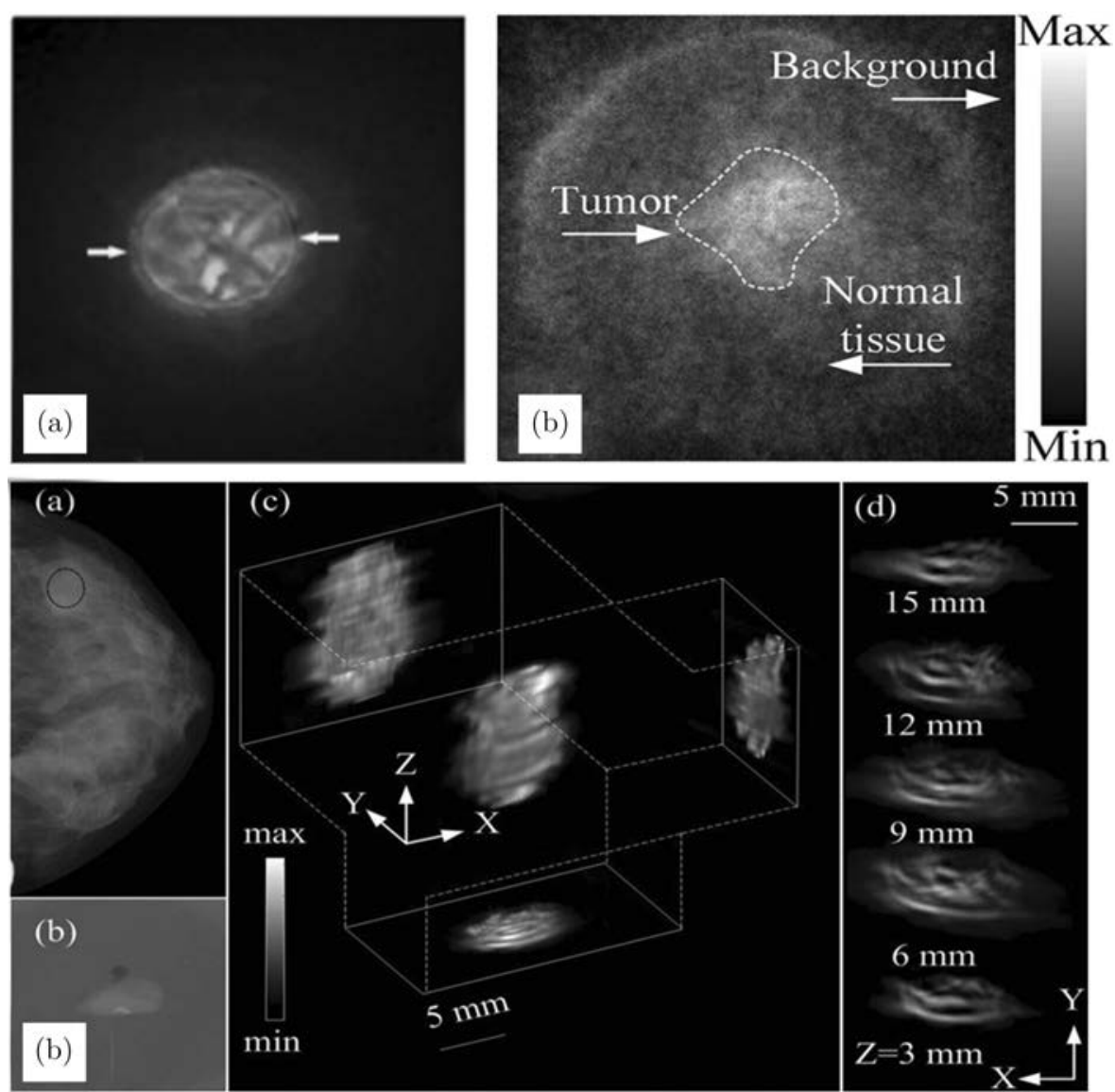

Fig. 12. The results of TAI of breast tumor. (a) Malignant breast tissue. ${ }^{83}$ (b) The pathology model composed of excised human breast tumor and the excised breast of an adult ewe. ${ }^{91}$ (c) Three-dimensional TAI experiment on breast model. ${ }^{93}$

clearly showed and conformed to histology slides in Fig. 13. Moreover, the values of TAI in select regions of the peripheral zone were associated with the disease status of the three histological specimens, and it can be seen that the strength of TA signal decreases with tumor involvement from specimens. Conclusion can be drawn that TAI can be a potential detection method for prostate cancer in the future.

\subsection{Foreign body detection}

The normal biological tissue dominated by water has a high dielectric loss factor and absorbs microwave

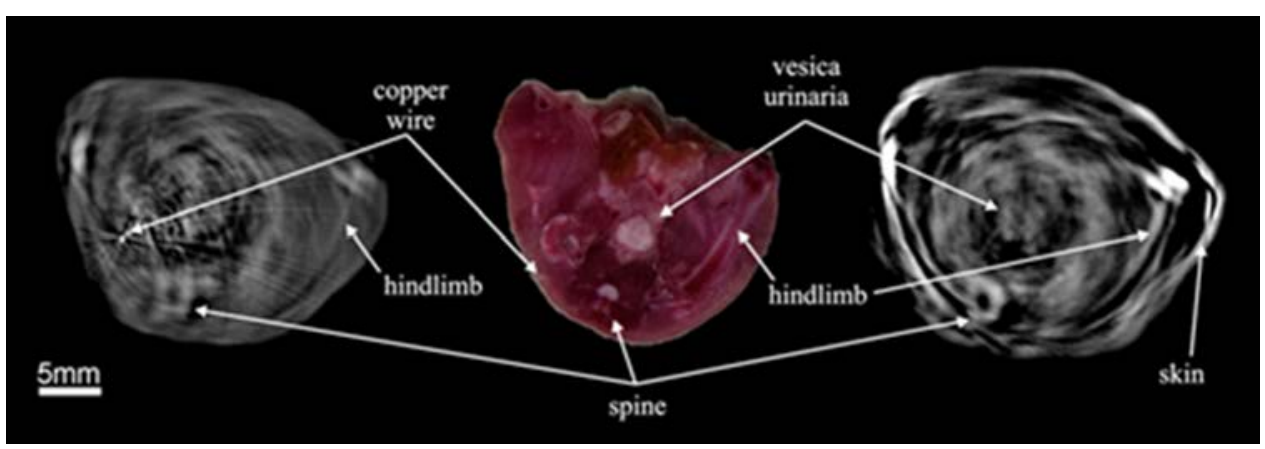

Fig. 13. Mouse imaging experiments ex vivo. (a) Cross-sectional TAI acquired from an intact mouse inserted with a $230 \mu \mathrm{m}$ diameter copper wire. (b) The histological slice of the mouse made at approximately the same level. (c) The similar TAI acquired from the mouse without the presence of the copper wire. ${ }^{35}$ 


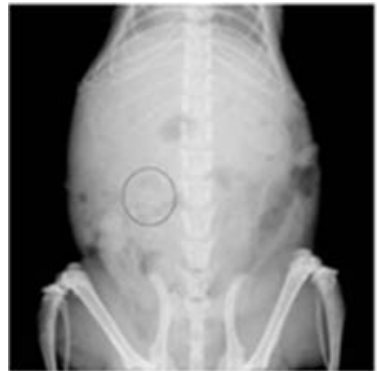

(a)

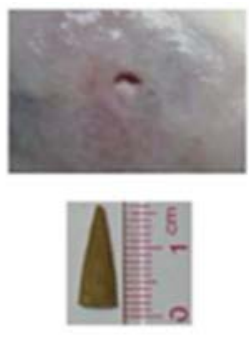

(b)

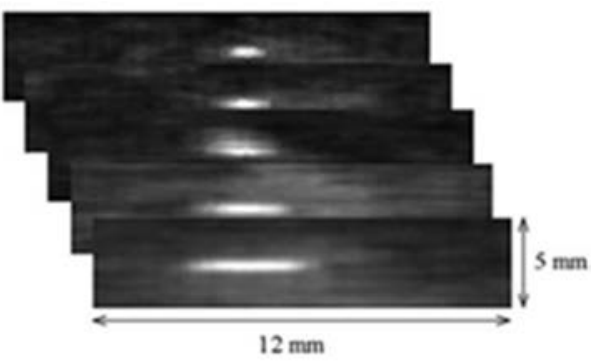

(c)

Fig. 14. Comparison of foreign body detection between TAI and X-ray imaging. (a) X-ray imaging of the living mouse with the bamboo. (b) Photographs of the mouse and the bamboo strip. (c) Five TA images along the vertical direction of the bamboo strip. ${ }^{84}$

strongly, whereas foreign bodies such as glass fiber, wood and bamboo with much less water content have much lower dielectric loss factors than the surrounding normal tissue. Therefore, TAI based on the differences of their electromagnetic properties has the potential to differentiate and image foreign body lesions. A TAI system by Xing et al. was developed to detect foreign targets in small animals. Figure 14(a) shows the X-ray imaging of the living mouse with the bamboo hidden in the body as marked in the dashed circle, and Fig. 14(b) shows the photographs of the inserted traumatic area of the mouse and the bamboo strip, respectively. Figure 14(c) shows the five TAI results along the vertical direction of the bamboo strip. Comparison with X-ray imaging and ultrasound imaging, the TAI can fill the blind zone for the X-ray band and may become an ideal and low cost modality for radiolucent foreign body detection and imaging in humans and animals. In addition, Arbabian and coworkers ${ }^{99}$ have achieved remote interrogation of highly dispersive media for deeply embedded abnormalities in 2015. This performance could enable a myriad of applications including medical imaging applications, security screening and nondestructive testing.

\subsection{Small animal imaging}

Besides cancer imaging and foreign body detection, TAI is also suitable for small animal imaging. Figure 15 shows the TAI results of a mouse reported by Ntziachristos et al. Figure 15(a) depicts reconstructed images from the mouse of the inserted line, and Fig. 15(b) depicts a photograph of the mouse slice obtained from the mice after frozen and cry
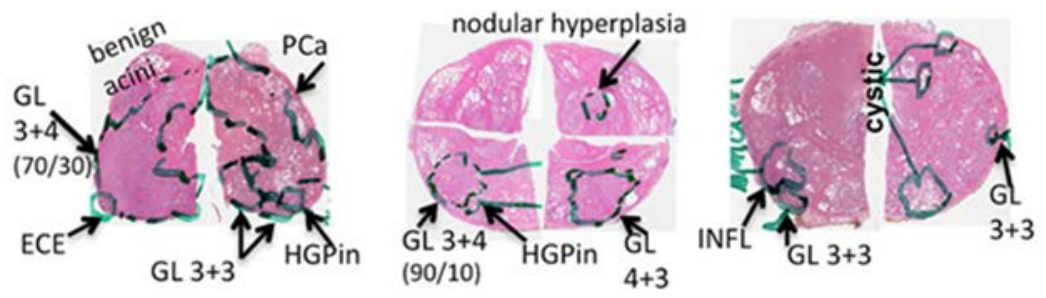

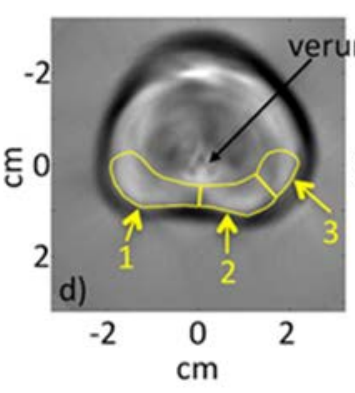

(a)

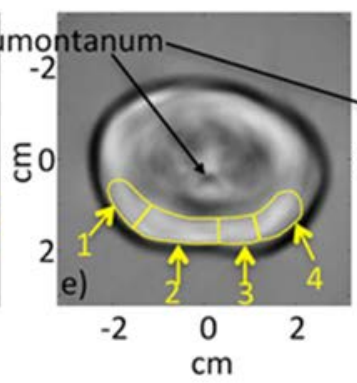

(b)

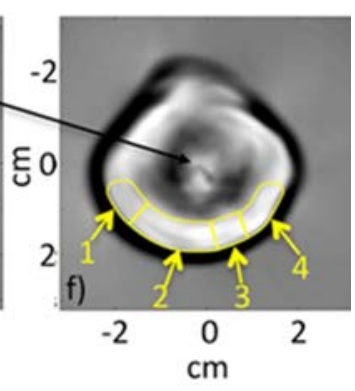

(c)

Fig. 15. (a-c) Three pathological sections of verumontanum. (d-f) The corresponding TAI reconstructions. ${ }^{31}$ 

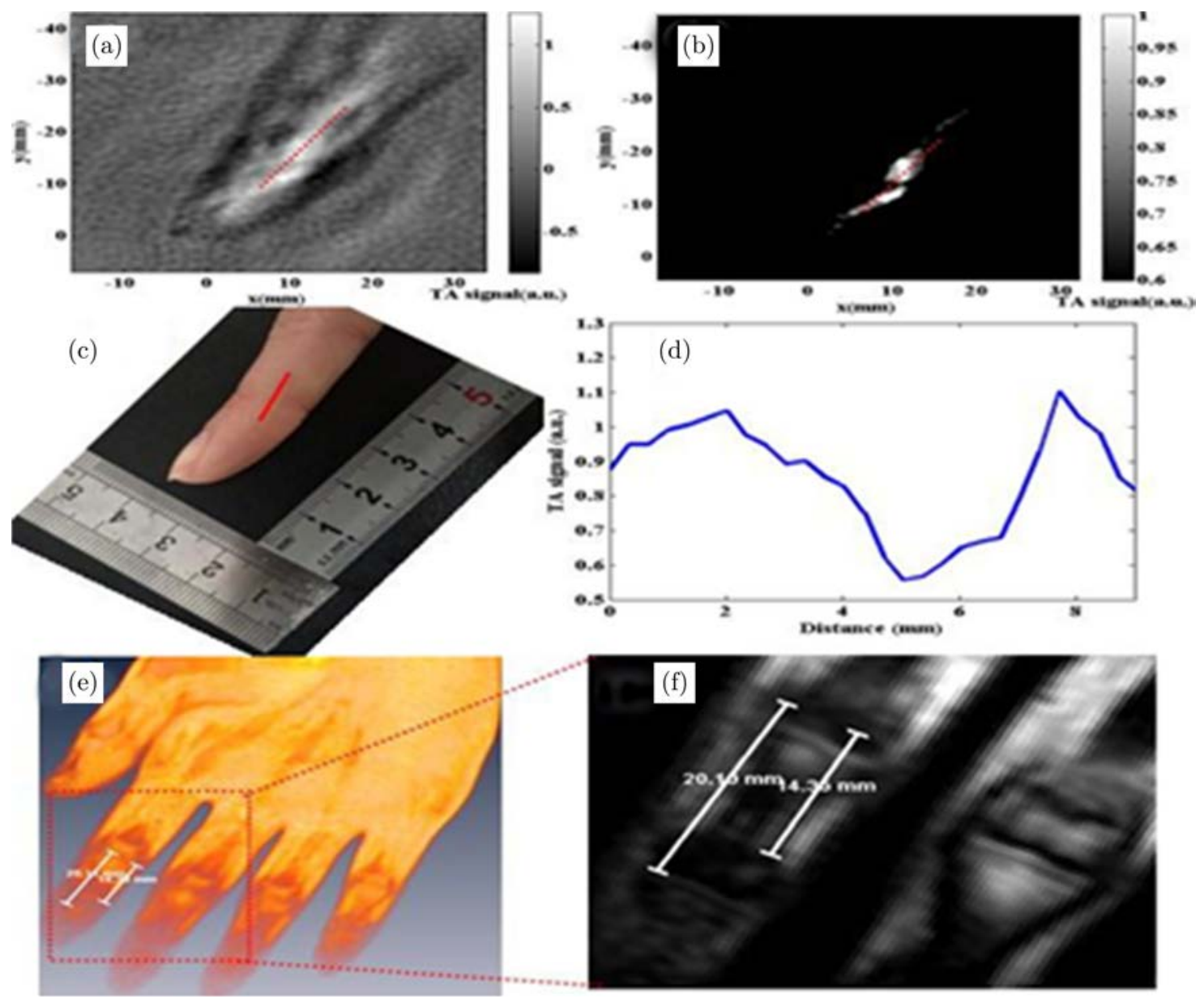

Fig. 16. (a) The TAI result of index finger image of female volunteer. (b) The TAI result by setting the threshold of (a). (c) The photograph of the index finger. (d) The recovered relative microwave absorption in red line. (e) The MRI results of the whole hand. (e) and (f) The TAI results corresponding to (e). ${ }^{43}$

sliced. It can be seen from the results that the copper wire on the left side of the spinal cord can be clearly detected. For comparison, Fig. 15(c) is obtained from the same mouse but without copper wire; therefore, it further shows anatomical details such as the spinal cord, the skin, the hindlimb and the urinary bladder, correlating well with the histological slice, such as in Fig. 15(b).

\subsection{Joint imaging and brain imaging}

As microwave absorption differences between the joint cavity and bone, joint imaging of TAI is possible. Jiang et al. proposed a TAI method to explore joint imaging, as shown in Fig. 16. The experimental results showed that the authors successfully detected joints and bones with TAI by demonstrating feasibility with three volunteers. Besides, Jiang et al. took an initial step to study the feasibility of TAI toward imaging human brain. Several phantom experiments were conducted to demonstrate that it is indeed possible to use TAI for noninvasive imaging of hemorrhagic stroke with high contrast. These two applications represent the latest research in TAI. Of particular note is that the brain imaging in humans is difficult due to the thick skull, and the problem has to be solved in the future.

\section{Prospects and Conclusions}

In summary, under the efforts of the researchers, the excitation source, data acquisition system and applications of TAI have been intensively studied. It is believed that TAI has great potential applications in both biomedical research and clinical study. The most possible research directions in the future are the following: 
1. Expand the application range: TAI has made great progress in imaging of breast cancer. Besides, TAI on prostate cancer has been reported. It indicates that TAI has certain advantages in cancer detection because the cancer tissue has relatively strong microwave absorption; therefore, expanding the application range to other cancers is rationale. Meanwhile, the examples of joint and brain imaging indicate that TAI has the potential to imaging damage of soft tissue, promising to be a new noninvasive functional imaging method.

2. Develop multi-modal imaging: TAI can map microwave absorption coefficient of biological tissues. However, the single-parameter imaging lacks other information and cannot meet the needs of getting more information of the body, which limits its availability. Therefore, in order to make doctors understand the internal structure of the body more accurately and comprehensively, combining TAI with other imaging modes (priority PAI and ultrasonic imaging) to recover the shortcomings of existing technology has practical significance and is urgently needed.

\section{Acknowledgments}

We thank Yi Luo, Baohua Qin, Yaping Li, Xin Wang, Yan Zhao and Wanling Luo for collecting references. This research is supported by the National Natural Science Foundation of China (61627827, 61331001, 81630046, 91539127), the Science and Technology Planning Project of Guangdong Province, China (2015B020233016, 2014B020215003, 2014A020215031, 2017A020215135), the Distinguished Young Teacher Project in Higher Education of Guangdong, China (YQ2015049), the Science and Technology Youth Talent for Special Project of Guangdong, China (2015TQ01X882), Young Teachers Scientific Research Cultivating Fund of South China Normal University (16KJ05) and China Postdoctoral Science Foundation (2017M610533).

\section{References}

1. R. A. Kruger, D. R. Reinecke, G. A. Kruger, "Thermoacoustic computed tomography - Technical considerations," Med. Phys. 26, 1832-1837 (1999).
2. R. A. Kruger, K. D. Miller, H. E. Reynolds, W. L. Kiser, D. R. Reinecke, G. A. Kruger, "Breast cancer in vivo: Contrast enhancement with thermoacoustic CT at $434 \mathrm{MHz}$ - Feasibility study," Radiology 216, 279-283 (2000).

3. R. A. Kruger, W. L. Kiser, D. R. Reinecke, G. A. Kruger, K. D. Miller, "Thermoacoustic molecular imaging of small animals," Mol. Imag. 2, 113-123 (2003).

4. L. V. Wang, X. Zhao, H. Sun, G. Ku, "Microwaveinduced acoustic imaging of biological tissues," Rev. Sci. Instrum. 70, 3744-3748 (1999).

5. G. Ku, L. V. Wang, "Scanning thermoacoustic tomography in biological tissue," Med. Phys. 27, 1195-1202 (2000).

6. D. Feng, Y. Xu, G. Ku, L. V. Wang, "Microwaveinduced thermoacoustic tomography: Reconstruction by synthetic aperture," Med. Phys. 28, 24272431 (2001).

7. G. Ku, L. V. Wang, "Scanning microwave-induced thermoacoustic tomography: Signal, resolution, and contrast," Med. Phys. 28, 4-10 (2001).

8. M. Xu, G. Ku, L. V. Wang, "Microwave-induced thermoacoustic tomography using multi-sector scanning," Med. Phys. 28, 1958-1963 (2001).

9. Y. Xu, L. V. Wang, "Signal processing in scanning thermoacoustic tomography in biological tissues," Med. Phys. 28, 1519-1524 (2001).

10. M. Xu, L. V. Wang, "Pulsed-microwave-induced thermoacoustic tomography: Filtered backprojection in a circular measurement configuration," Med. Phys. 29, 1661-1669 (2002).

11. M. Xu, L. V. Wang, "Time-domain reconstruction for thermoacoustic tomography in a spherical geometry," IEEE Trans. Med. Imag. 21, 814-822 (2002).

12. Y. Xu, D. Feng, L. V. Wang, "Exact frequencydomain reconstruction for thermoacoustic tomography. I. Planar geometry," IEEE Trans. Med. Imag. 21, 823-828 (2002).

13. Y. Xu, M. Xu, L. V. Wang, "Exact frequencydomain reconstruction for thermoacoustic tomography. II. Cylindrical geometry," IEEE Trans. Med. Imag. 21, 829-833 (2002).

14. M. Xu, Y. Xu, L. V. Wang, "Time-domain reconstruction algorithms and numerical simulations for thermoacoustic tomography in various geometries," IEEE Trans. Biomed. Eng. 50, 1086-1099 (2003).

15. Y. Xu, L. V. Wang, "Effects of acoustic heterogeneity in breast thermoacoustic tomography," IEEE Trans. Ultrason. Ferroelectr. Freq. Control 50, 1134-1146 (2003).

16. Y. $\mathrm{Xu}, \mathrm{L} . \mathrm{V}$. Wang, G. Ambartsoumian, P. Kuchment, "Reconstructions in limited-view thermoacoustic tomography," Med. Phys. 31, 724-733 (2004). 
17. G. Ku, B. D. Fornage, X. Jin, M. Xu, K. K. Hunt, L. V. Wang, "Thermoacoustic and photoacoustic tomography of thick biological tissues toward breast imaging," Technol. Cancer Res. Treat. 4, 559-565 (2005).

18. X. Jin, Y. Xu, L. V. Wang, Y. R. Fang, C. I. Zanelli, S. M. Howard, "Imaging of high-intensity focused ultrasound-induced lesions in soft biological tissue using thermoacoustic tomography," Med. Phys. 32, 5-11 (2005).

19. X. Jin, L. V. Wang, "Thermoacoustic tomography with correction for acoustic speed variations," Phys. Med. Biol. 51, 6437-6488 (2006).

20. Y. Xu, L. V. Wang, "Rhesus monkey brain imaging through intact skull with thermoacoustic tomography," IEEE Trans. Ultrason. Ferroelectr. Freq. Control 53, 542-548 (2006).

21. X. Jin, C. Li, L. V. Wang, "Effects of acoustic heterogeneities on transcranial brain imaging with microwave-induced thermoacoustic tomography," Med. Phys. 35, 3205-3214 (2008).

22. C. Li, M. Pramanik, G. Ku, L. V. Wang, "Image distortion in thermoacoustic tomography caused by microwave diffraction," Phys. Rev. E 77, 031923 (2008).

23. M. Pramanik, G. Ku, C. Li, L. V. Wang, "Design and evaluation of a novel breast cancer detection system combining both thermoacoustic (TA) and photoacoustic (PA) tomography," Med. Phys. 35, 2218-2223 (2008).

24. Y. Xie, B. Guo, J. Li, G. Ku, L. V. Wang, "Adaptive and robust methods of reconstruction (ARMOR) for thermoacoustic tomography," IEEE Trans. Biomed. Eng. 55, 2741-2752 (2008).

25. M. Pramanik, G. Ku, L. V. Wang, "Tangential resolution improvement in thermoacoustic and photoacoustic tomography using a negative acoustic lens," J. Biomed. Opt. 14, 024-028 (2009).

26. Z. Liu, L. Liu, Y. Xu, L. V. Wang, "Transcranial thermoacoustic tomography: A comparison of two imaging algorithms," IEEE Trans. Med. Imag. 32, 289-294 (2013).

27. Y. He, C. Liu, L. Lin, L. V. Wang, "Comparative effects of linearly and circularly polarized illumination on microwave induced thermoacoustic tomography," IEEE Antenn. Wirel. Propag. Lett. (2016). doi: 10.1109/LAWP.2017.2652853.

28. S. K. Patch, "Thermoacoustic tomography Consistency conditions and the partial scan problem," Phys. Med. Biol. 49, 2305-2315 (2004).

29. A. T. Eckhart, R. T. Balmer, W. A. See, S. K. Patch, "Ex vivo thermoacoustic imaging over large fields of view with $108 \mathrm{MHz}$ irradiation," IEEE Trans. Biomed. Eng. 58, 2238-2246 (2011).
30. D. Fallon, L. Yan, G. W. Hanson, S. K. Patch, "RF testbed for thermoacoustic tomography," Rev. Sci. Instrum. 80, 064301 (2009).

31. S. K. Patch, D. Hull, M. Thomas, S. K. Griep, K. Jacobsohn, W. A. See, "Thermoacoustic contrast of prostate cancer due to heating by very high frequency irradiation," Phys. Med. Biol. 60, 689-708 (2015).

32. S. K. Patch, D. Hull, W. A. See, G. W. Hanson, "Toward quantitative whole organ thermoacoustics with a clinical array plus one very low-frequency channel applied to prostate cancer imaging," IEEE Trans. Ultrason. Ferroelectr. Freq. Control 63, 245-255 (2016).

33. S. K. Patch, M. K. Covo, A. Jackson, "Thermoacoustic range verification using a clinical ultrasound array provides perfectly co-registered overlay of the Bragg peak onto an ultrasound image," Phys. Med. Biol. 61, 5621 (2016).

34. D. Razansky, S. Kellnberger, V. Ntziachristos, "Near-field radiofrequency thermoacoustic tomography with impulse excitation," Med. Phys. 37, 4602-4607 (2010).

35. S. Kellnberger, A. Hajiaboli, D. Razansky, V. Ntziachristos, "Near-field thermoacoustic tomography of small animals," Phys. Med. Biol. 56, 3433-3444 (2011).

36. M. Omar, S. Kellnberger, G. Sergiadis, D. Razansky, V. Ntziachristos, "Near-field thermoacoustic imaging with transmission line pulsers," Med. Phys. 39, 4460-4466 (2012).

37. A. Hajiaboli, S. Kellnberger, V. Ntziachristos, D. Razansky, "Design and time-domain analysis of a high-voltage impulsed test-bed for near-field thermoacoustic tomography," Prog. Electromagn. Res. 139, 105-119 (2013).

38. L. Yao, G. Guo, H. Jiang, "Quantitative microwaveinduced thermoacoustic tomography," Med. Phys. 37, 3752-3759 (2010).

39. L. Huang, L. Yao, L. Liu, R. Jian, H. Jiang, "Quantitative thermoacoustic tomography: Recovery of conductivity maps of heterogeneous media," Appl. Phys. Lett. 101, 244106 (2012).

40. L. Huang, J. Rong, L. Yao, W. Qi, D. Wu, J. Xu, H. Jiang, "Quantitative thermoacoustic tomography for ex vivo imaging conductivity of breast tissue," Chin. Phys. Lett. 30, 124301 (2013).

41. Z. Chi, Y. Zhao, L. Huang, Z. Zheng, H. Jiang, "Thermoacoustic imaging of rabbit knee joints," Med. Phys. 43, 6226-6233 (2016).

42. L. Huang, W. Cai, Y. Zhao, "In vivo tumor detection with combined MR-photoacoustic-thermoacoustic imaging," J. Innov. Opt. Health Sci. 9, 1650015 (2016). 
43. L. Huang, W. Qi, Y. Zhao, Z. Chi, N. Zhang, J. Rong, D. Lai, "Thermoacoustic imaging of human finger joints and bones," X Acoust. Imaging Sens. 1, 28-31 (2015).

44. D. R. Bauer, X. Wang, J. Vollin, H. Xin, R. S. Witte, "Spectroscopic thermoacoustic imaging of water and fat composition," Appl. Phys. Lett. 101, 033705 (2012).

45. X. Wang, D. R. Bauer, J. L. Vollin, D. G. Manzi, R. S. Witte, H. Xin, "Impact of microwave pulses on thermoacoustic imaging applications," IEEE Antenn. Wirel. Propag. Lett. 11, 1634-1637 (2012).

46. X. Wang, D. R. Bauer, R. Witte, H. Xin, "Microwave-induced thermoacoustic imaging model for potential breast cancer detection," IEEE Trans. Biomed. Eng. 59, 2782-2791 (2012).

47. T. Qin, X. Wang, Y. Qin, P. Ingram, G. Wan, R. S. Witte, H. Xin, "Experimental validation of a numerical model for thermoacoustic imaging applications," IEEE Antenn. Wirel. Propag. Lett. 14, 1235-1238 (2015).

48. T. Qin, X. Wang, Y. Qin, G. Wan, R. S. Witte, H. Xin, "Quality improvement of thermoacoustic imaging based on compressive sensing," IEEE Antenn. Wirel. Propag. Lett. 14, 1200-1203 (2015).

49. X. Wang, T. Qin, R. S. Witte, H. Xin, "Computational feasibility study of contrastenhanced thermoacoustic imaging for breast cancer detection using realistic numerical breast phantoms," IEEE Trans. Microw. Theory Tech. 63, 1489-1501 (2015).

50. H. Nan, A. Arbabian, "Stepped-frequency continuouswave microwave-induced thermoacoustic imaging," Appl. Phys. Lett. 104, 224104 (2014).

51. M. Aliroteh, H. Nan, A. Arbabian, "Microwaveinduced thermoacoustic tomography for subcutaneous vascular imaging," IEEE Ultrason. Symp. (IUS), pp. 1948-5727 (2016). DOI: 10.1109/ ULTSYM.2016.7728643.

52. X. Feng, F. Gao, Y. Zheng, "Magnetically mediated thermoacoustic imaging toward deeper penetration," Appl. Phys. Lett. 103, 083704 (2013).

53. X. Feng, F. Gao, Y. Zheng, "Modulatable magnetically mediated thermoacoustic imaging with magnetic nanoparticles," Appl. Phys. Lett. 106, 153702 (2015).

54. G. Fei, X. Feng, Y. Zheng, "Advanced photoacoustic and thermoacoustic sensing and imaging beyond pulsed absorption contrast," J. Opt. 18, 074006 (2016).

55. F. Gao, Y. Zheng, X. Feng, C. Ohl, "Thermoacoustic resonance effect and circuit modelling of biological tissue," Appl. Phys. Lett. 102, 063702 (2013).

56. G. Zangerl, O. Scherzer, M. Haltmeier, "Circular integrating detectors in photo and thermoacoustic tomography," Inverse Probl. Sci. Eng. 17, 133-142 (2009).

57. M. Agranovsky, P. Kuchment, "Uniqueness of reconstruction and an inversion procedure for thermoacoustic and photoacoustic tomography with variable sound speed," Inverse Probl. 23, 2089-2102 (2007).

58. M. Haltmeier, O. Scherzer, P. Burgholzer, R. Nuster, G. Paltauf, "Thermoacoustic tomography and the circular radon transform: Exact inversion formula," Math. Mod. Meth. Appl. Sci. 17, 635-655 (2007).

59. K. H. Lim, J. H. Lee, Q. H. Liu, "Thermoacoustic tomography forward modeling with the spectral element method," Med. Phys. 35, 4-12 (2008).

60. A. Mashal, J. H. Booske, S. C. Hagness, "Toward contrast-enhanced microwave-induced thermoacoustic imaging of breast cancer: An experimental study of the effects of microbubbles on simple thermoacoustic targets," Phys. Med. Biol. 54 641-650 (2009).

61. D. Passeri, U. Sassi, A. Bettucci, E. Tamburri, F. Toschi, S. Orlanducci, M. L. Terranova, M. Rossi, "Thermoacoustic emission from carbon nanotubes imaged by atomic force microscopy," Adv. Funct. Mater. 22, 2956-2963 (2012).

62. N. A. Rejesh, H. Pullagurla, M. Pramanik, M. Pramanik, "Deconvolution-based deblurring of reconstructed images in photoacoustic/thermoacoustic tomography," J. Opt. Soc. Am. A $\mathbf{3 0}$, 1994-2001 (2013).

63. M. Pramanik, "Improving tangential resolution with a modified delay-and-sum reconstruction algorithm in photoacoustic and thermoacoustic tomography," J. Opt. Soc. Am. A 31, 621-627 (2014).

64. O. Ogunlade, P. Beard, "Exogenous contrast agents for thermoacoustic imaging: An investigation into the underlying sources of contrast," Med. Phys. 42, 170-181 (2015).

65. C. Zhang, Y. Wang, "A reconstruction algorithm for thermoacoustic tomography with compensation for acoustic speed heterogeneity," Phys. Med. Biol. 53, 4971-4982 (2008).

66. X. Zhu, Z. Zhao, J. Wang, G. Chen, Q. Liu, "Active adjoint modeling method in microwave induced thermoacoustic tomography for breast tumor," IEEE Trans. Biomed. Eng. 61, 1957-1966 (2014).

67. H. Peng, J. Hu, C. Hu, "Fe3O4@ MnO2@ PAA nanoparticles for magnetically targeted microwavethermal therapy guided by thermoacoustic imaging," J. Biomater. Tissue Eng. 6, 12-19 (2016).

68. A. Dewantari, S. Y. Jeon, S. Kim, "Analysis of microwave-induced thermoacoustic signal generation using computer simulation," J. Electromagn. Eng. Sci. 16, 1-6 (2016). 
69. E. Hernández-Rosales, E. Cedeño, J. HernandezWong, "Thermoacoustic and thermoreflectance imaging of biased integrated circuits: Voltage and temperature maps," Appl. Phys. Lett. 109, 041902 (2016).

70. S. Liu, Z. Zhao, X. Zhu, Z.-L. Wang, J. Song, B. Wang, Y.-B. Gong, Z.-P. Nie, Q. H. Liu, "Analysis of short pulse impacting on microwave induced thermo-acoustic tomography," Prog. Electromagn. Res. C 61, 37-46 (2016).

71. B. M. Mihiretie, D. Cederkrantz, M. Sundin, "Thermal depth profiling of materials for defect detection using hot disk technique," AIP $A d v$. 6, 085217 (2016).

72. Y. He, Y. Shen, C. Liu, "Suppressing excitation effects in microwave induced thermoacoustic tomography by multi-view Hilbert transformation," Appl. Phys. Lett. 110, 053701 (2017).

73. S. Islam, S. Mahmud, M. Biglarbegian, "Effect of magnetic field on the Nusselt number of a multiplate thermoacoustic system," Int. J. Therm. Sci. 108, 145-158 (2016).

74. Y. Wu, Z. Tang, Y Chi, "A simultaneous multiprobe detection label-free optical-resolution photoacoustic microscopy technique based on microcavity transducer," J. Innov. Opt. Health Sci. 6, 1350027 (2013).

75. Y. Zhao, S. Yang, "Photoacoustic viscoelasticity imaging of biological tissues with intensitymodulated continuous-wave laser," J. Innov. Opt. Health Sci. 6, 1350033 (2013).

76. J. Zhong, S. Yang, "Contrast-enhanced photoacoustic imaging using indocyanine green-containing nanoparticles," J. Innov. Opt. Health Sci. 7, 1350029 (2014).

77. B. Yan, H. Qin, "Indocyanine green loaded graphene oxide for high-efficient photoacoustic tumor therapy," J. Innov. Opt. Health Sci. 9, 1642001 (2016).

78. B. Yin, D. Xing, Y. Wang, Y. Zeng, Y. Tan, Q. Chen, "Fast photoacoustic imaging system based on 320-element linear transducer array," Phys. Med. Biol. 49, 1339-1346 (2004).

79. D. Yang, D. Xing, H. Gu, Y. Tan, "Fast multielement phase-controlled photoacoustic imaging based on limited-field filtered back projection algorithm," Appl. Phys. Lett. 87, 194101 (2005).

80. L. Zeng, D. Xing, H. Gu, D. Yang, S. Yang, L. Xiang, "Fast microwave-induced thermoacoustic tomography based on multi-element phasecontrolled focus technique," Chin. Phys. Lett. 23, 1215-1218 (2006).

81. L. Zeng, D. Xing, H. Gu, D. Yang, S. Yang, L. Xiang, "High antinoise photoacoustic tomography based on a modified filtered backprojection algorithm with combination wavelet," Med. Phys. 34, 556-563 (2007).

82. L. Nie, D. Xing, D. Yang, L. Zeng, Q. Zhou, "Detection of foreign body using fast thermoacoustic tomography with a multielement linear transducer array," Appl. Phys. Lett. 90, 174109 (2007).

83. L. Nie, D. Xing, Q. Zhou, D. Yang, H. Guo, "Microwave-induced thermoacoustic scanning CT for high-contrast and noninvasive breast cancer imaging," Med. Phys. 35, 4026-4032 (2008).

84. L. Nie, D. Xing, S. Yang, "In vivo detection and imaging of low-density foreign body with microwave-induced thermoacoustic tomography," Med. Phys. 36, 3429-3437 (2009)

85. C. Lou, D. Xing, "Temperature monitoring utilizing thermoacoustic signals during pulsed microwave thermotherapy: A feasibility study," Int. J. Hypertherm. 26, 338-346 (2010).

86. C. Lou, L. Nie, D. Xu, "Effect of excitation pulse width on thermoacoustic signal characteristics and the corresponding algorithm for optimization of imaging resolution," J. Appl. Phys. 110, 083101 (2011).

87. C. Cao, L. Nie, C. Lou, D. Xing, "The feasibility of using microwave-induced thermoacoustic tomography for detection and evaluation of renal calculi," Phys. Med. Biol. 55, 5203-5212 (2010).

88. C. Lou, S. Yang, Z. Ji, Q. Chen, D. Xing, "Ultrashort microwave-induced thermoacoustic imaging: A breakthrough in excitation efficiency and spatial resolution," Phys. Rev. Lett. 109, 218101 (2012).

89. Y. Fu, Z. Ji, W. Ding, F. Ye, C. Lou, "Thermoacoustic imaging over large field of view for three-dimensional breast tumor localization: A phantom study," Med. Phys. 41, 110701 (2014).

90. W. Ding, C. Lou, J. Qiu, Z. Zhao, Q. Zhou, M. Liang, S. Yang, Z. Ji, D. Xing, "Targeted Fefilled carbon nanotube as a multifunctional contrast agent for thermoacoustic and magnetic resonance imaging of tumor in living mice," Nanomed. Nanotechnol. Biol. Med. 12, 235-244 (2016).

91. F. Ye, Z. Ji, W. Ding, C. Lou, S. Yang, D. Xing, "Ultrashort microwave-pumped real-time thermoacoustic breast tumor imaging system," IEEE Trans. Med. Imag. 35, 839-844 (2016).

92. W. Ding, Z. Ji, F. Ye, C. Lou, D. Xing, "Near-field microwave distribution measurement with a point detector base on thermoacoustic effect," IEEE Trans. Microw. Theory Tech. 63, 3272-3276 (2015).

93. Z. Ji, C. Lou, S. Yang, D. Xing, "Three-dimensional thermoacoustic imaging for early breast cancer detection," Med. Phys. 39, 6738-6744 (2012). 
94. Z. Ji, W. Ding, F. Ye, C. Lou, D. Xing, "Shapeadapting thermoacoustic imaging system based on flexible multi-element transducer," Appl. Phys. Lett. 107, 094104 (2015).

95. Z. Ji, C. Lou, Y. Shi, W. Ding, S. Yang, D. Xing, "A microwave detection way by electromagnetic and elastic resonance: Breaking the bottleneck of spatial resolution in microwave imaging," Appl. Phys. Lett. 107, 164103 (2015).

96. Z. Ji, W. Ding, S. Yang, Q. Chen, D. Xing, "Remote measurement of microwave distribution based on optical detection," Appl. Phys. Lett. 108, 014104 (2016).
97. Z. Ji, W. Ding, F. Hao, C. Lou, "Handheld thermoacoustic scanning system based on a linear-array transducer," Ultrason. Imag. 38, 276-284 (2016)

98. Z. Ji, Y. Fu, S. Yang, "Microwave-induced thermoacoustic imaging for early breast cancer detection," J. Innov. Opt. Health Sci. 06, 1350001 (2013).

99. H. Nan, K. C. Boyle, N. Apte, M. S. Aliroteh, A. Bhuyan, A. Nikoozadeh, B. T. Khuri-Yakub, A. Arbabian, "Non-contact thermoacoustic detection of embedded targets using airborne-capacitive micromachined ultrasonic transducers," Appl. Phys. Lett. 106, 084101 (2015). 